Supplementary Information

\title{
In Situ Decoration of Ultrafine Ru Nanocrystals on N-Doped Graphene \\ Tube and Their Applications Oxygen Reduction and Hydrogen \\ Evolution Catalyst
}

Barun Kumar Barman, ${ }^{\ddagger}$ Bidushi Sarkar, ${ }^{\ddagger}$ Priyadarshini Ghosh, ${ }^{\ddagger}$ Monalisa Ghosh, ${ }^{\perp}$ Gowravaram

Mohan Rao, ${ }^{\perp}$ Karuna Kar Nanda ${ }^{\ddagger *}$

${ }^{*}$ Materials Research Centre, Indian Institute of Science, Bangalore-560012

$\perp$ Department of Instrumentation and Applied Physics, Indian Institute of Science, Bangalore-560012, India

*Corresponding author: Email: nanda@iisc.ac.in

${ }^{\S}$ Authors contributed equally. 


\section{Calibration of reference electrodes and conversion to RHE}

The calibration of reference electrodes $(\mathrm{Hg} / \mathrm{HgO}$ and $\mathrm{Ag} / \mathrm{AgCl})$ were performed in a standard three-electrode cell with two Pt wires act as both the working and counter electrodes, and the $\mathrm{Hg} / \mathrm{HgO}$ or $\mathrm{Ag} / \mathrm{AgCl}$ electrode as the reference electrode. Cyclic voltammetry $(\mathrm{CV})$ is then run at a scan rate of $5 \mathrm{mV} / \mathrm{s}$ in alkaline solution. The potential at which the current crossed zero is the thermodynamic potential for the hydrogen electrode reactions. For $\mathrm{Ag} / \mathrm{AgCl}$ reference electrode in $0.5 \mathrm{M} \mathrm{H}_{2} \mathrm{SO}_{4}$ solution, the zero current point is at $-0.225 \mathrm{~V}$, so $\mathrm{E}(\mathrm{RHE})=\mathrm{E}(\mathrm{Ag} / \mathrm{AgCl})+0.225 \mathrm{~V}$. For $\mathrm{Hg} / \mathrm{HgO}$ electrode in $1 \mathrm{M} \mathrm{KOH}$ solution, the zero current point is at $-0.98 \mathrm{~V}$, so $\mathrm{E}(\mathrm{RHE})=\mathrm{E}(\mathrm{Hg} / \mathrm{HgO})+$ $0.98 \mathrm{~V}$.
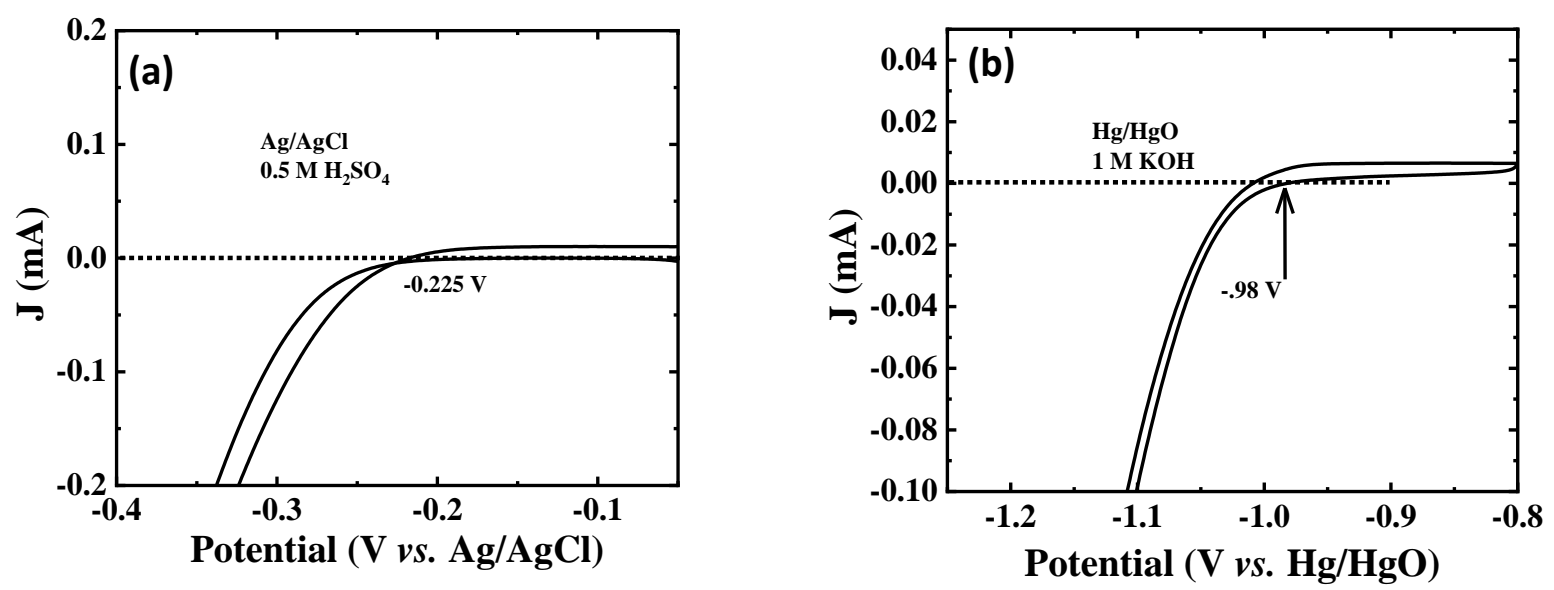

Figure $\mathrm{S} 1$ ( $\mathrm{a}$ and $\mathrm{b}$ ) $\mathrm{Ag} / \mathrm{AgCl}$ and $\mathrm{Hg} / \mathrm{HgO}$ reference electrode calibration curve in $0.5 \mathrm{M} \mathrm{H}_{2} \mathrm{SO}_{4}$ medium and $1 \mathrm{M} \mathrm{KOH}$ medium, respectively. 

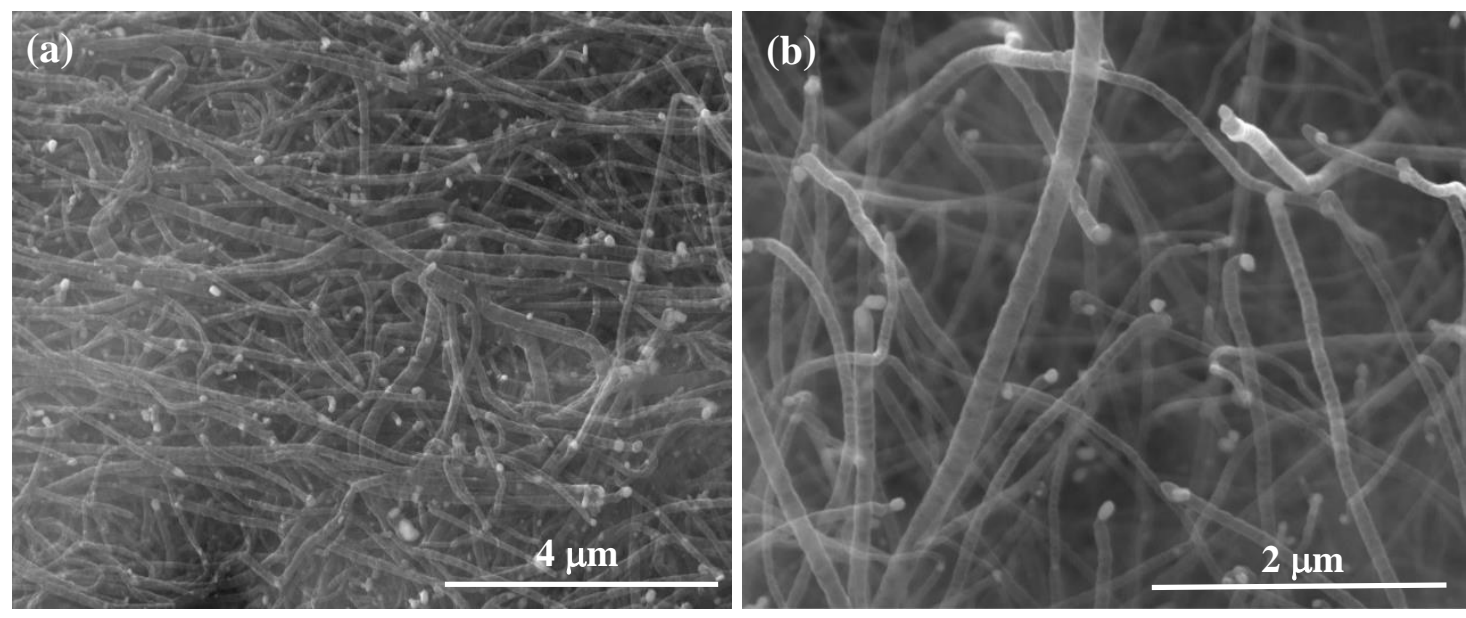

Figure S2 ( $a$ and $b)$ SEM image of NGT

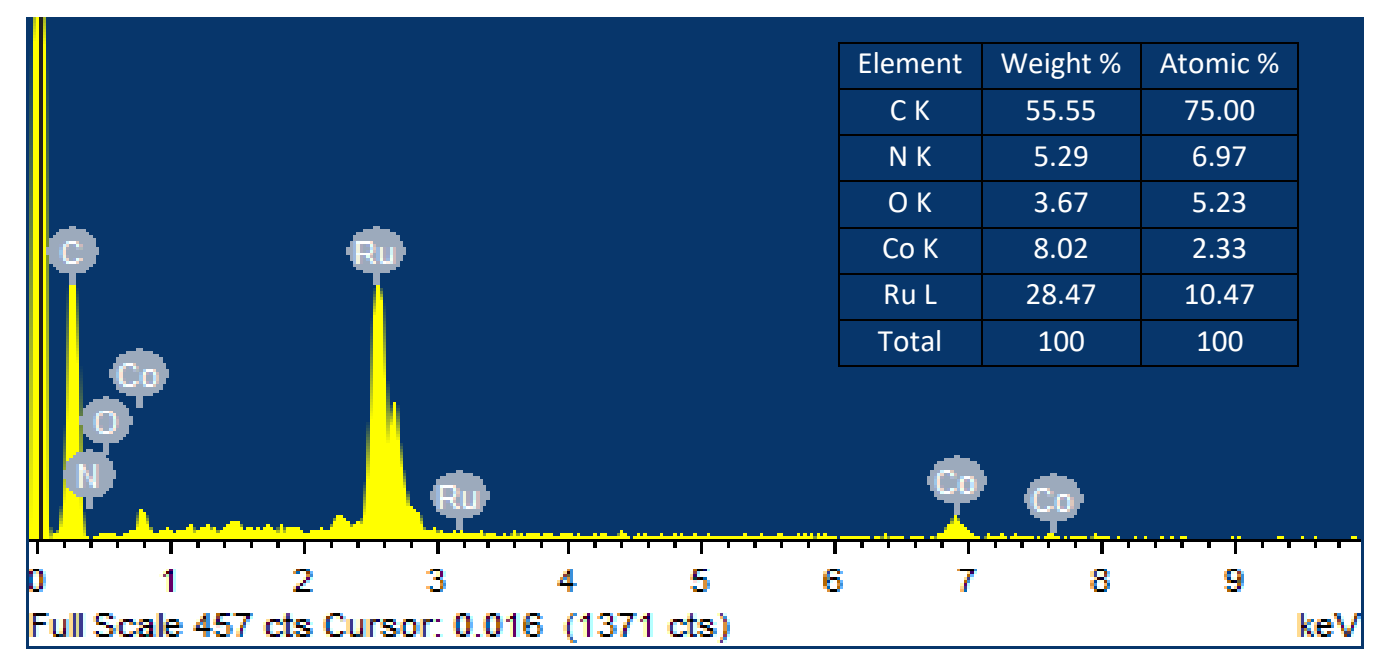

Figure S3 EDX spectra of Ru@NGT (inset: element composition table) 

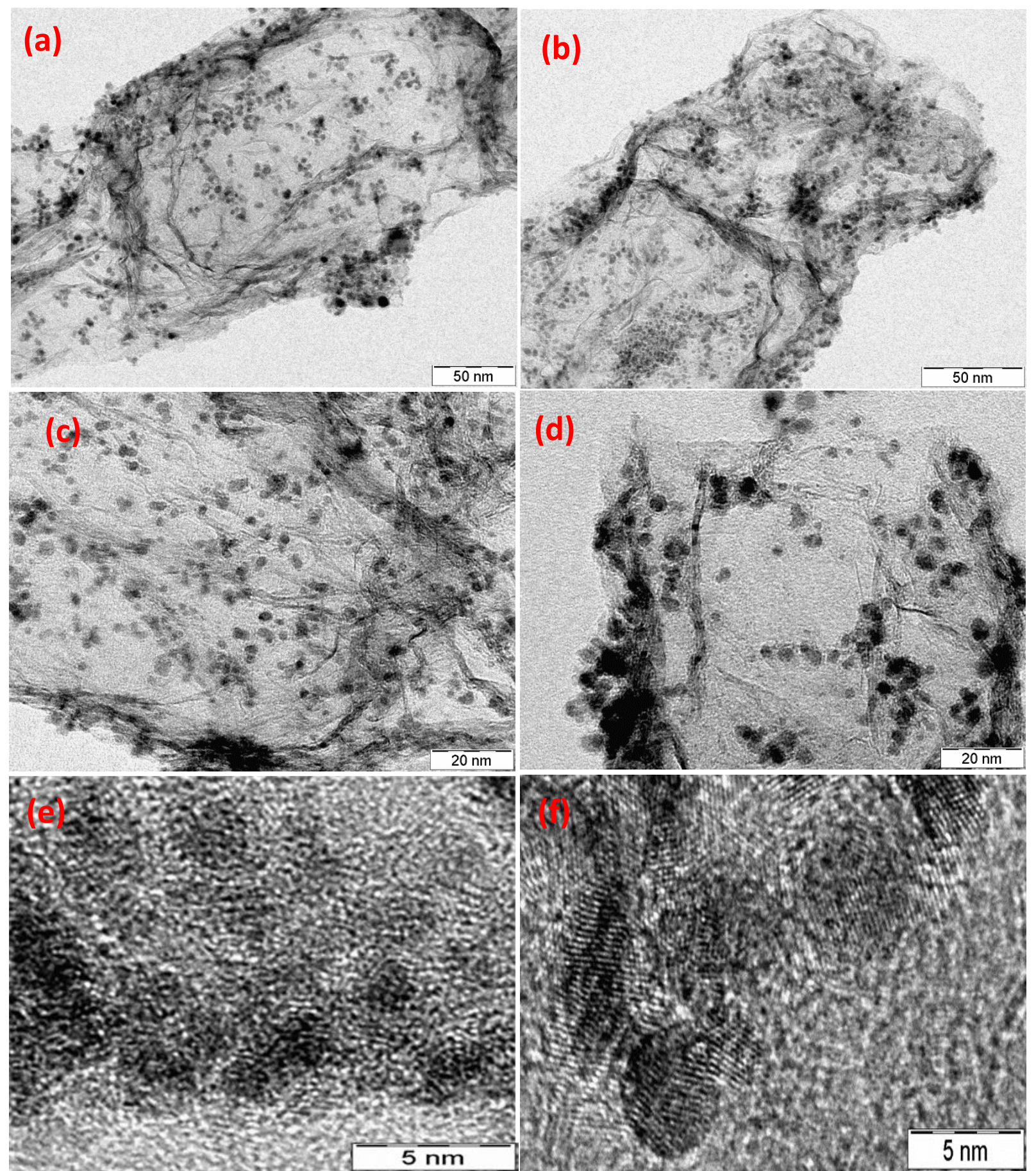

Figure S4 (a-d) TEM, and (e, f) HRTEM images of ultrafine Ru@NGT. 


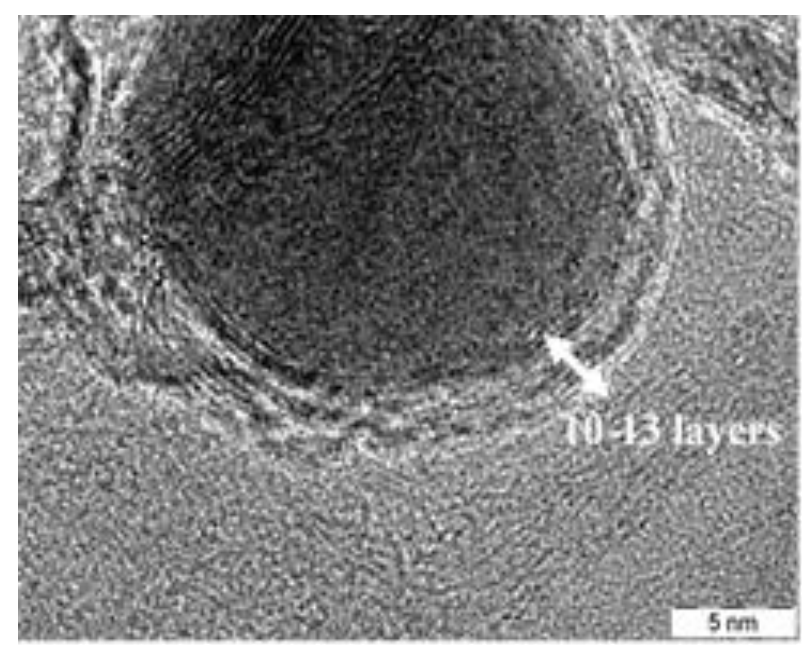

Figure S5 HRTEM image of NGT
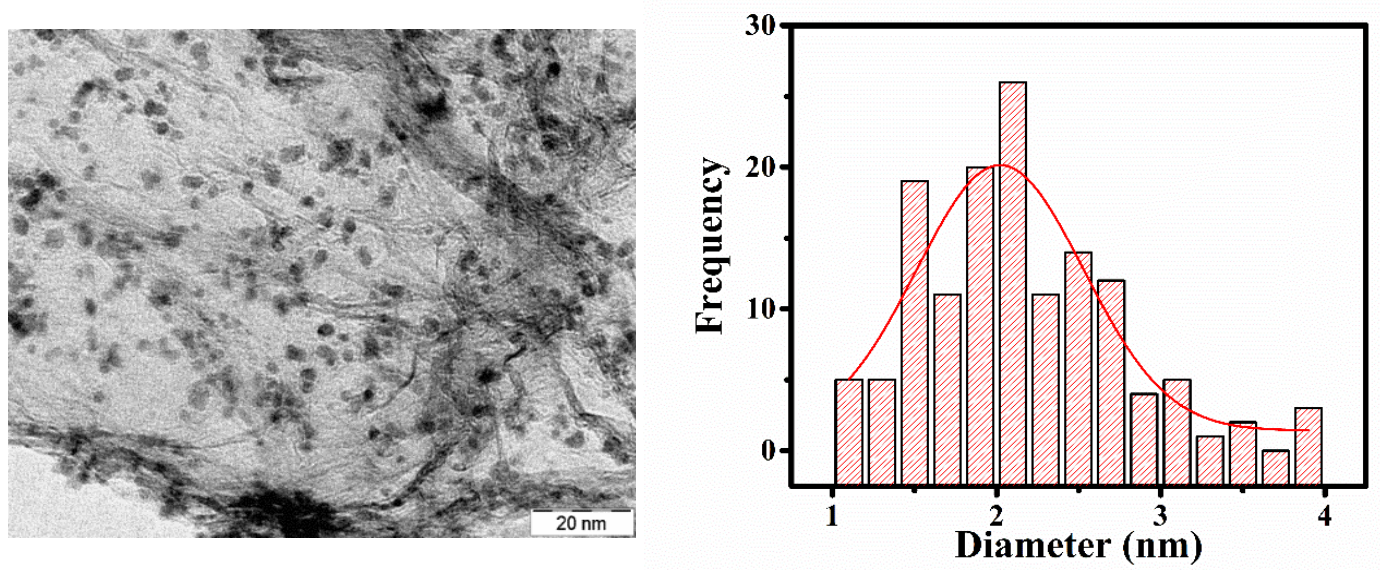

Figure S6 TEM image and particle size distribution of Ru@NGT. 

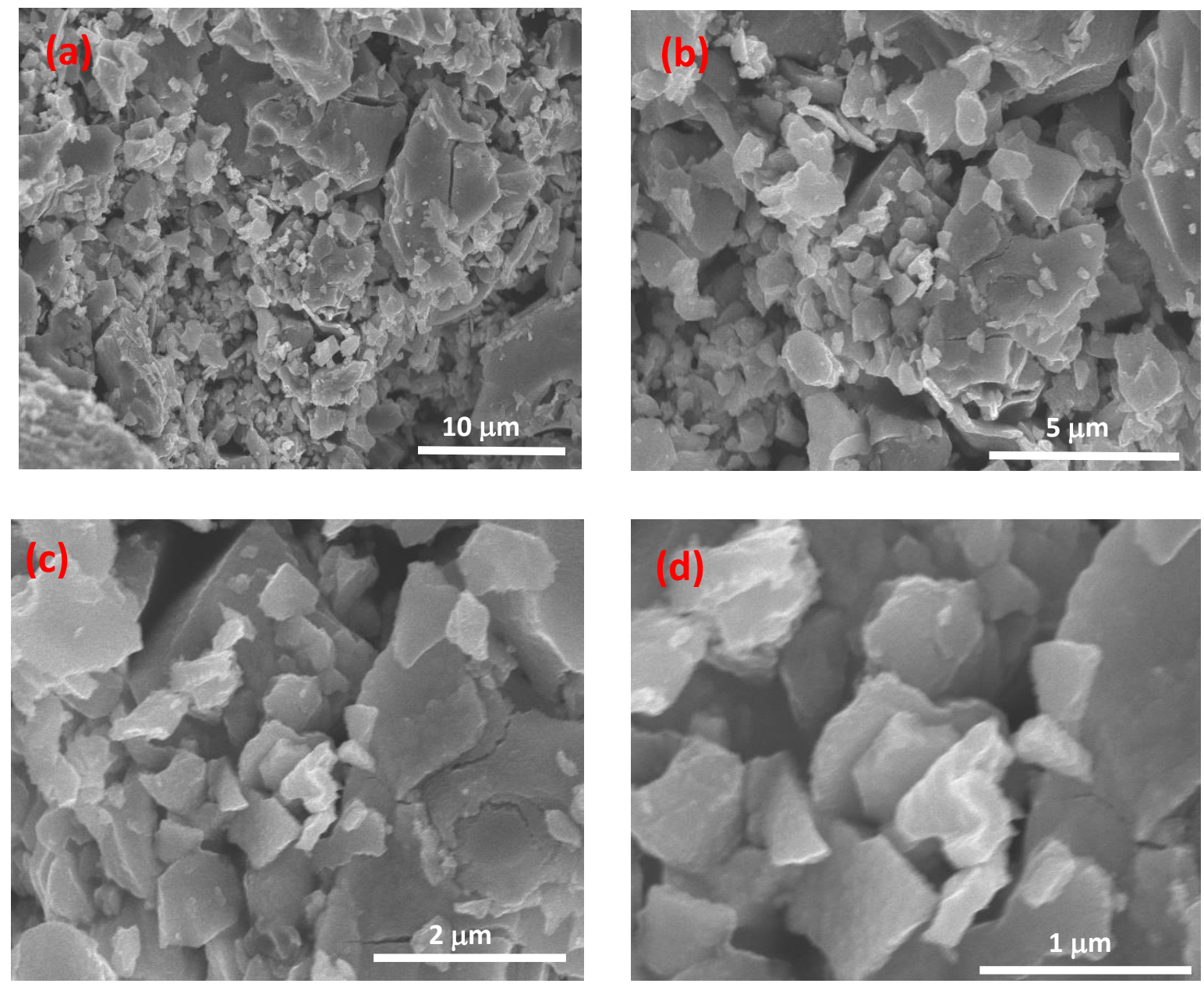

Figure S7 (a to d) SEM images of irregular Ru nanostructures. 

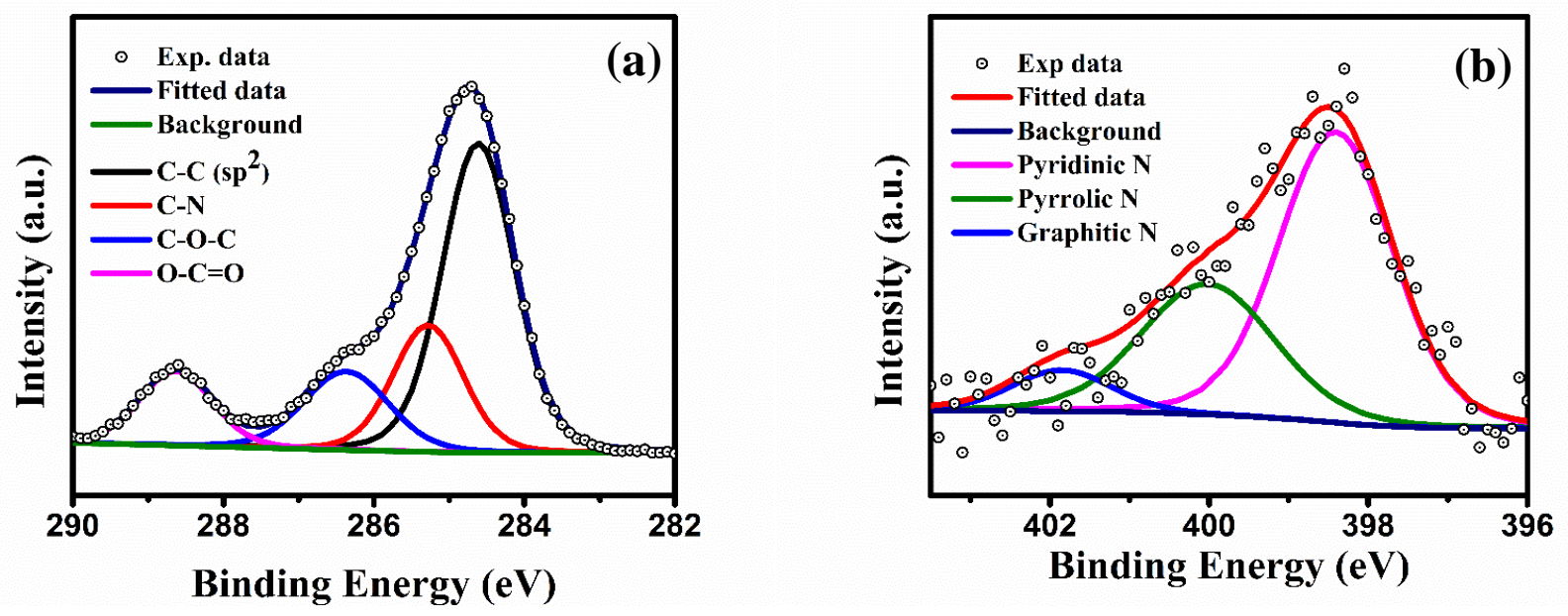

Figure S8 HRXPS of (a) C 1s, and (b) N 1s for NGT

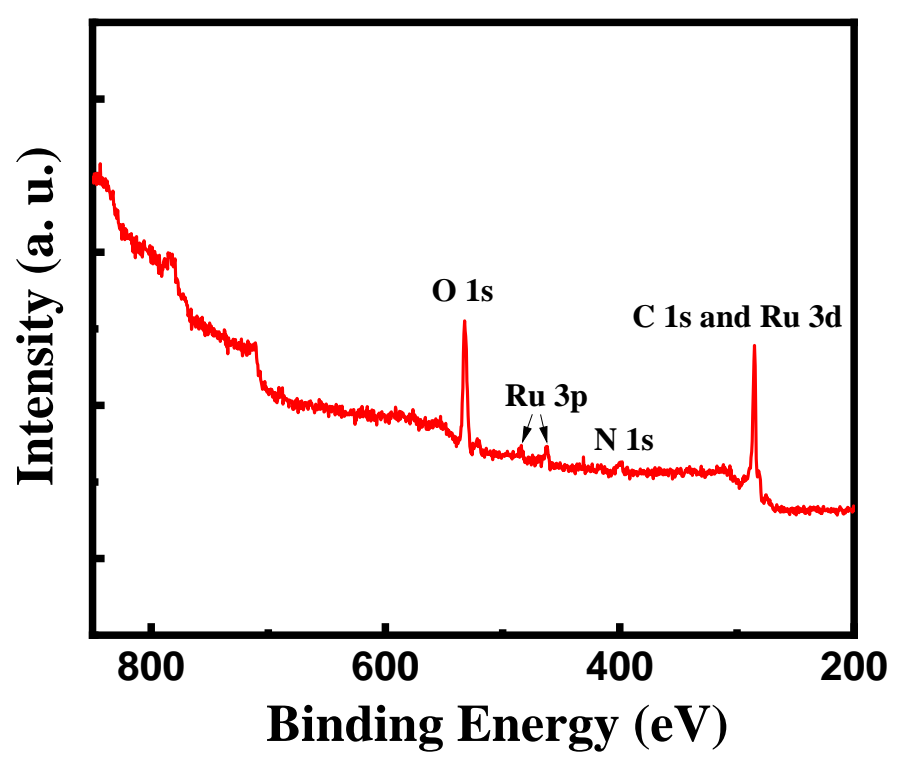

Figure S9 XPS survey spectrum of Ru@NGT 


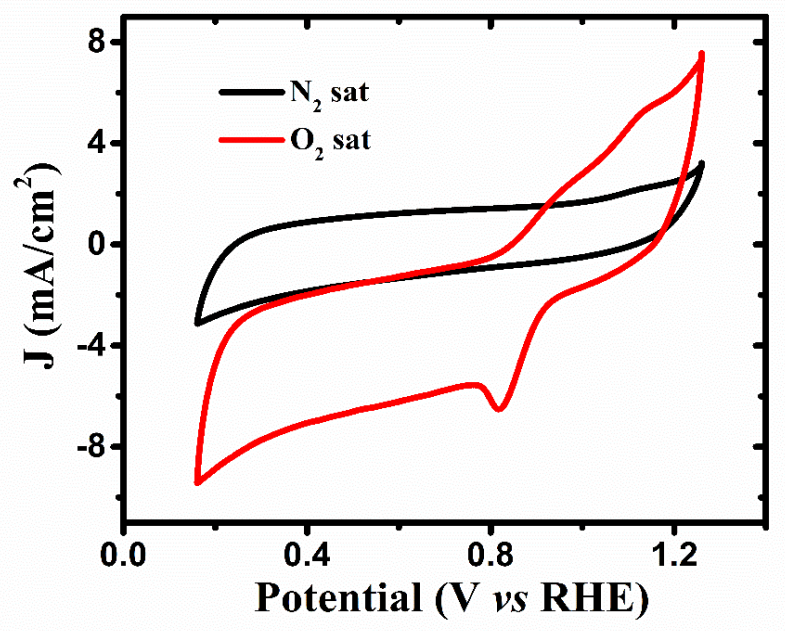

Figure $\mathrm{S} 10 \mathrm{CV}$ of Ru@NGT in $\mathrm{N}_{2}$ and $\mathrm{O}_{2}$ saturated solution with $0.1 \mathrm{M} \mathrm{KOH}$ at a scan rate of 50 $\mathrm{mV} / \mathrm{s}$ with $600 \mathrm{rpm}$.
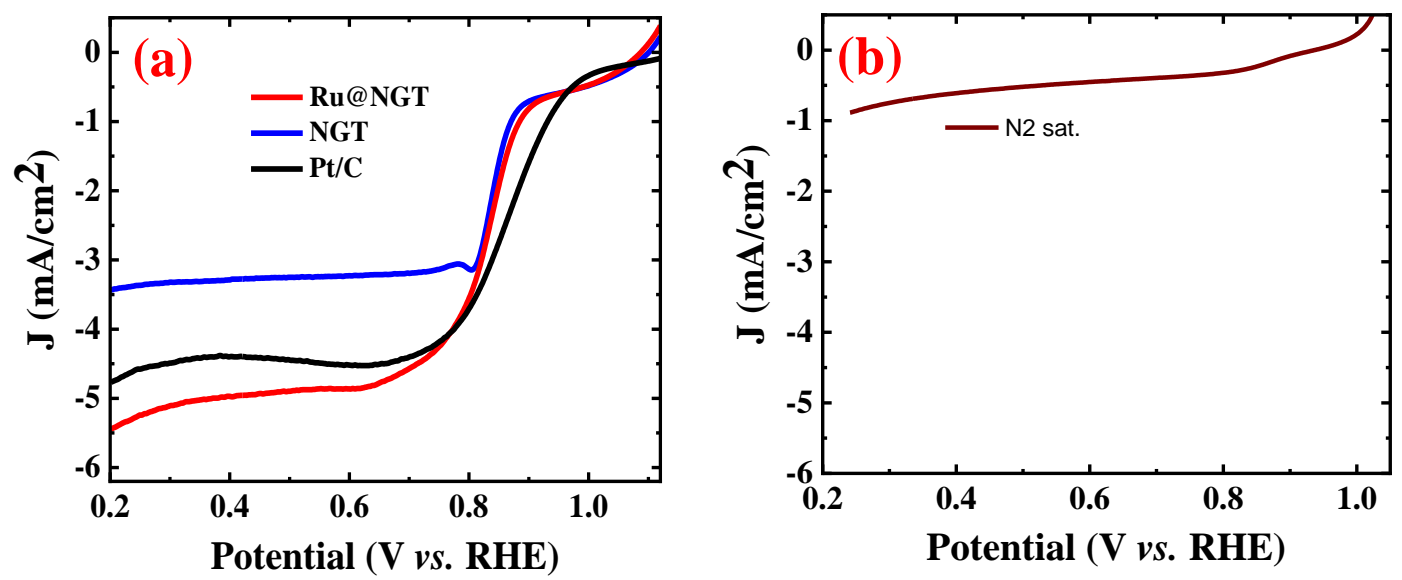

Figure S11 (a) ORR comparison spectra of different catalyst in $0.1 \mathrm{M} \mathrm{KOH}$ solution with rotation speed $1400 \mathrm{rpm}$ with scan rate $10 \mathrm{mV} / \mathrm{s}$. (b) LSV spectra in $\mathrm{N}_{2}$ saturated solution 


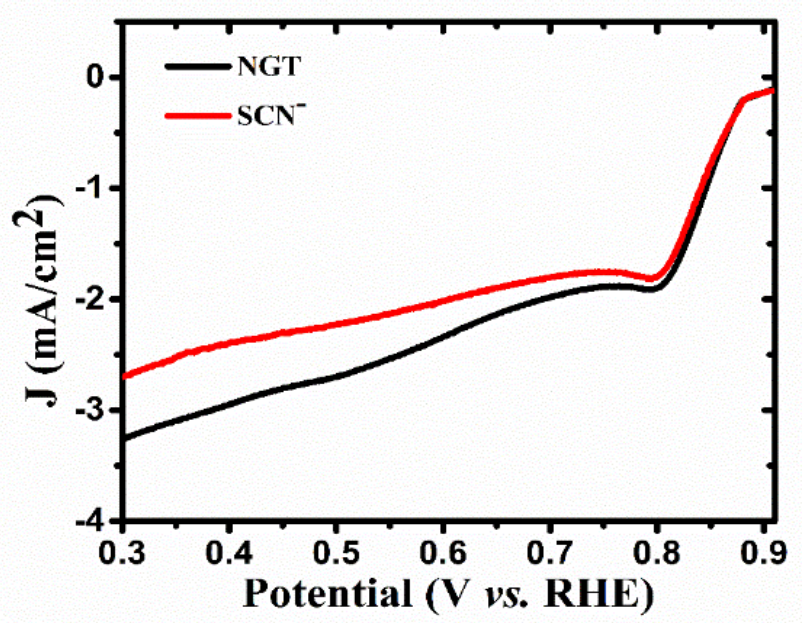

Figure S12 LSV of NGT before and after poisoning with $10 \mathrm{mM}$ of $\mathrm{NaSCN}$.

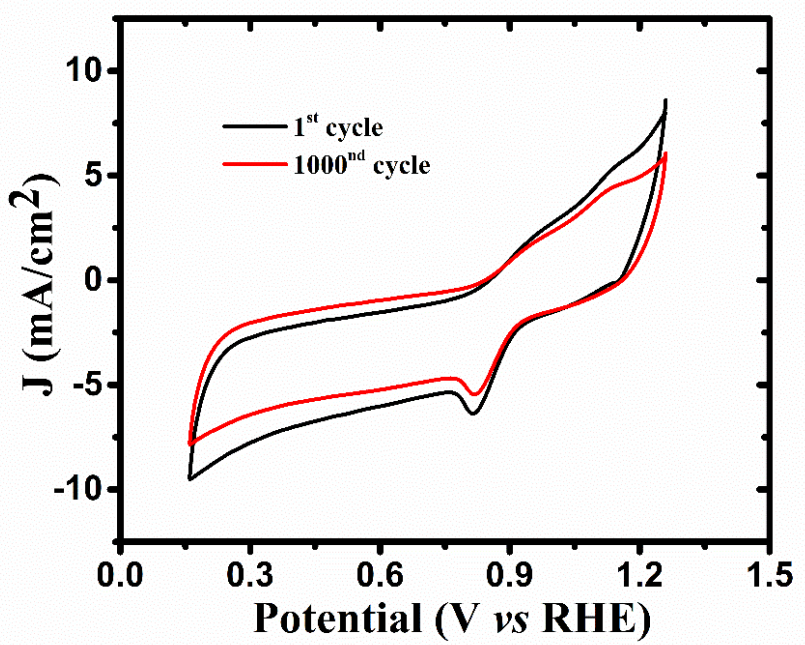

Figure S13 ORR stability plot of Ru@NGT up to $1000^{\text {nd }}$ cycle at a scan rate of $100 \mathrm{mV} / \mathrm{s}$. 

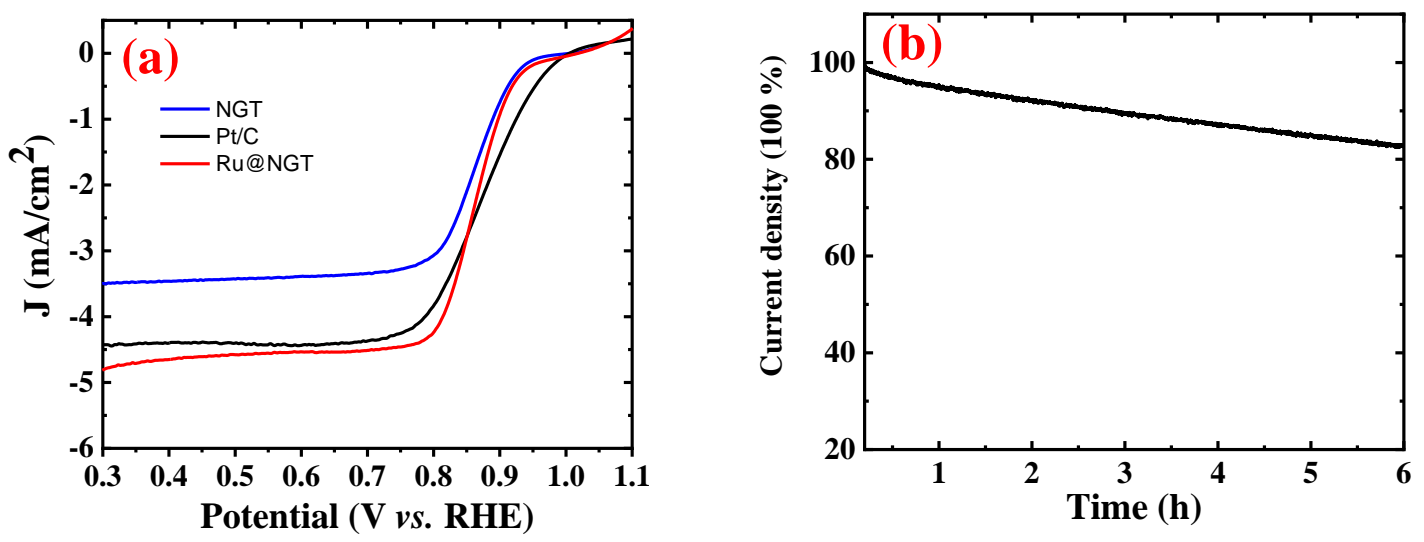

Figure S14 (a) LSV polarization of different catalyst and stability of Ru@NGT catalyst (Graphite rod as counter electrode and $\mathrm{Hg} / \mathrm{HgO}$ as reference electrode, scan rate $10 \mathrm{mV} / \mathrm{s}$, rotation speed $1400 \mathrm{rpm}$ in $0.1 \mathrm{M} \mathrm{KOH}$ solution).
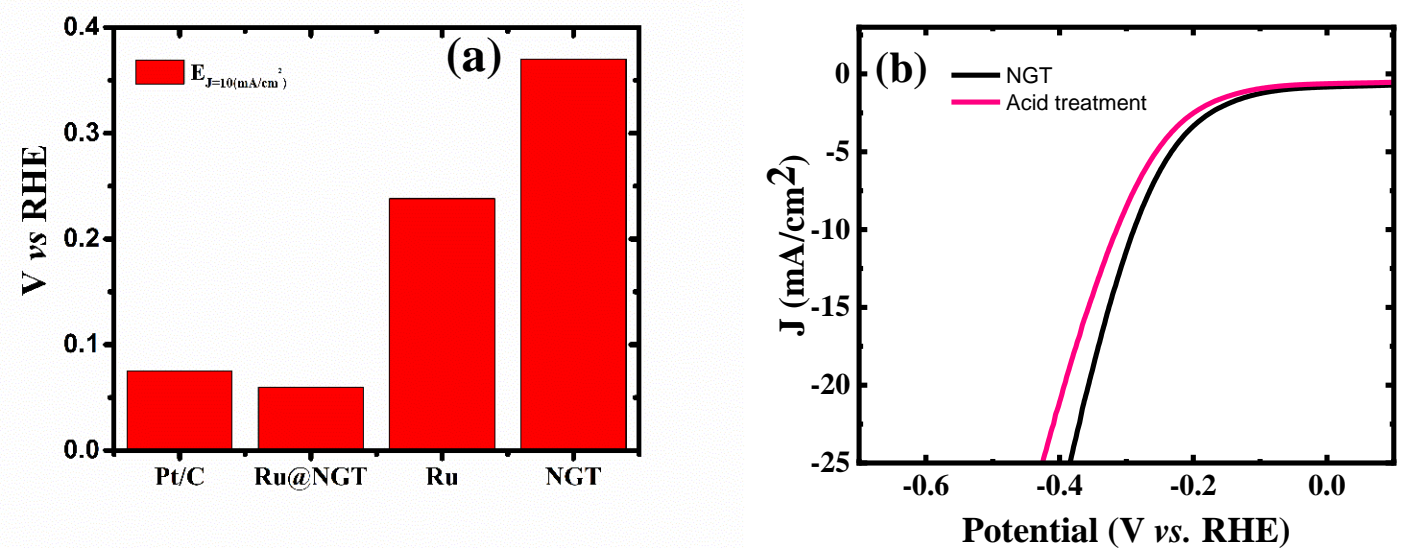

Figure S15 HER potential required to achieve $10 \mathrm{~mA} / \mathrm{cm}^{2}$ current density of different catalysts in $1 \mathrm{M} \mathrm{KOH}$ solution. (b) HER polarization curve of NGT and acid treated NGT. 


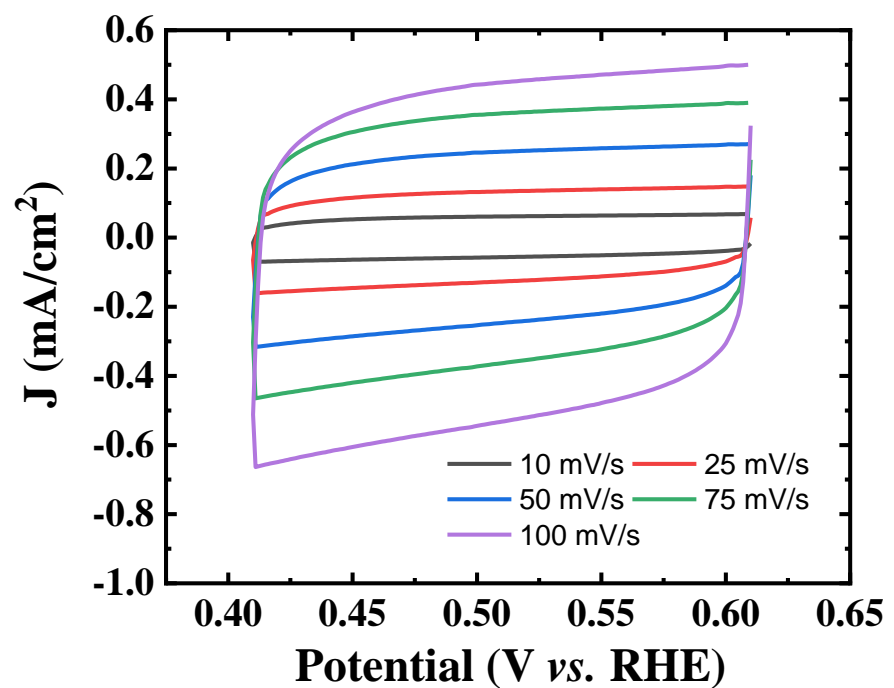

Figure S16 CV at different scan rates in the faradaic silent region of NGT

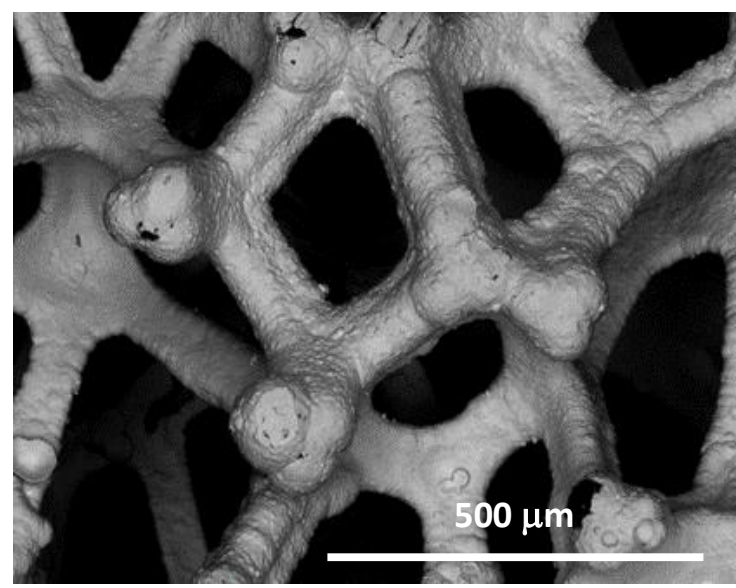

Figure S17 SEM image of Ni foam 
Table S1 Comparison table of ORR performance of Ru@NGT catalyst with other recently developed catalyst in alkaline media $(0.1 \mathrm{M} \mathrm{KOH})$

\begin{tabular}{|c|c|c|c|c|}
\hline Catalyst & $\begin{array}{l}\text { Loading } \\
\left(\mathrm{mg} / \mathrm{cm}^{2}\right)\end{array}$ & $\begin{array}{c}E_{\text {onset }} \\
\text { (V vs RHE) }\end{array}$ & $\begin{array}{c}E_{1 / 2} \\
\text { (V vs RHE) }\end{array}$ & Ref. \\
\hline $\begin{array}{c}\mathrm{Co}_{3} \mathrm{O}_{4} / \mathrm{Co} @ \mathrm{~N}-\mathrm{G}- \\
450\end{array}$ & - & 0.962 & 0.808 & 1 \\
\hline $\mathrm{Au}_{67} \mathrm{Pd}_{33} / \mathrm{CNs}$ & 80.8 & 0.94 & 0.83 & 2 \\
\hline $\begin{array}{c}\mathrm{CoO} @ \mathrm{Co}_{3} \mathrm{O}_{4} / \mathrm{NSG}- \\
650\end{array}$ & 0.24 & - & 0.79 & 3 \\
\hline $\mathrm{Fe} / \mathrm{N} / \mathrm{C}$ & 0.1 & 0.94 & 0.84 & 4 \\
\hline NPCA-900 & - & 0.93 & 0.80 & 5 \\
\hline Pd NPAs & - & 0.926 & 0.837 & 6 \\
\hline FePPyC-900 & 0.4 & 0.960 & 0.87 & 7 \\
\hline $\mathrm{CN}_{\mathrm{x}} / \mathrm{CMK}-3$ & 0.246 & 0.96 & 0.83 & 8 \\
\hline $\mathrm{N}-\mathrm{CSH}$ & 0.1 & 0.927 & 0.529 & 9 \\
\hline Co-NC & 0.21 & $\sim 0.9$ & 0.83 & 10 \\
\hline $\mathrm{Co}, \mathrm{N}-\mathrm{CNF}$ & 0.12 & 0.88 & - & 11 \\
\hline $\begin{array}{l}\text { Polydopamine - } \\
\text { encapsulated } \\
\mathrm{Fe}_{3}\left[\mathrm{Co}(\mathrm{CN})_{6}\right]_{2}\end{array}$ & 0.4 & 0.88 & 0.80 & 12 \\
\hline $\begin{array}{l}\text { Co/N-carbon } \\
\text { fibres }\end{array}$ & 0.306 & 0.95 & - & 13 \\
\hline NGPC/NCNT-900 & - & 0.91 & - & 14 \\
\hline $\mathrm{Fe}-\mathrm{N} / \mathrm{G}$ & - & 0.87 & - & 15 \\
\hline Ru@NGT & 0.428 & 0.97 & 0.83 & This work \\
\hline
\end{tabular}


Table S2 Comparison table of HER performance of Ru@NGT catalyst with other recently developed catalyst in alkaline media.

\begin{tabular}{|c|c|c|c|c|}
\hline Catalyst & $\begin{array}{l}\text { Loading } \\
\left(\mathrm{mg} / \mathrm{cm}^{2}\right)\end{array}$ & Electrolyte & $\begin{array}{l}E_{J=10 \mathrm{~mA} / \mathrm{cm} 2} \\
(\mathrm{mV})\end{array}$ & Ref. \\
\hline $\begin{array}{l}1 \mathrm{~T} \\
\mathrm{MoS}_{2} / \mathrm{Ni}^{2+\delta} \mathrm{O}_{\delta}(\mathrm{OH})_{2-\delta}\end{array}$ & 0.5 & $1 \mathrm{M} \mathrm{KOH}$ & 185 & 16 \\
\hline $\mathrm{o}-\mathrm{CoSe}_{2} \mid \mathrm{P}$ & 1.02 & $1 \mathrm{M} \mathrm{KOH}$ & 104 & 17 \\
\hline $\mathrm{MoS}_{2} / \mathrm{Co}(\mathrm{OH})_{2}$ & 0.285 & $1 \mathrm{M} \mathrm{KOH}$ & 128 & 18 \\
\hline W-SAC & 0.408 & $0.1 \mathrm{M} \mathrm{KOH}$ & 85 & 19 \\
\hline $\begin{array}{l}\mathrm{Cu} N \mathrm{ND} / \mathrm{Ni}_{3} \mathrm{~S}_{2} \mathrm{NTs}- \\
\mathrm{CFs}\end{array}$ & 0.52 & $1 \mathrm{M} \mathrm{KOH}$ & 128 & 20 \\
\hline MoP@C & 0.354 & $1 \mathrm{M} \mathrm{KOH}$ & 81 & 21 \\
\hline CoMos & 0.84 & $1 \mathrm{M} \mathrm{KOH}$ & 100 & 22 \\
\hline $\begin{array}{l}\text { Ultrathin } \mathrm{Ni} \\
\text { Nanosheets/ } \\
\text { Carbon cloth }\end{array}$ & 0.53 & $1 \mathrm{M} \mathrm{KOH}$ & 80 & 23 \\
\hline $\begin{array}{l}\mathrm{Ni}_{0.89} \mathrm{Co}_{0.11} \mathrm{Se}_{2} \\
\mathrm{MNSN} / \mathrm{NF}\end{array}$ & 2.62 & $1 \mathrm{M} \mathrm{KOH}$ & 85 & 24 \\
\hline $\begin{array}{l}\text { 3D core-shell } \\
\mathrm{Cu} @ \mathrm{NiFe} \text { LDH/CF }\end{array}$ & - & $1 \mathrm{M} \mathrm{KOH}$ & 116 & 25 \\
\hline $\begin{array}{l}\mathrm{N}, \mathrm{S} \text {-Carbon } \\
\text { nanosheets }\end{array}$ & - & $1 \mathrm{M} \mathrm{KOH}$ & 380 & 26 \\
\hline $\mathrm{Cu}_{2-\mathrm{x}} @ \mathrm{RuNPS}$ & 0.23 & $1 \mathrm{M} \mathrm{KOH}$ & 82 & 27 \\
\hline $\mathrm{Ru} / \mathrm{C}_{3} \mathrm{~N}_{4} / \mathrm{C}$ & - & $1 \mathrm{M} \mathrm{KOH}$ & 79 & 28 \\
\hline $1 \mathrm{D}-\mathrm{RuO}_{2}-\mathrm{CN}$ & 0.17 & $0.5 \mathrm{M} \mathrm{KOH}$ & 95 & 29 \\
\hline $\mathrm{NiSe} / \mathrm{NF}$ & 2.8 & $1 \mathrm{M} \mathrm{KOH}$ & 96 & 30 \\
\hline Ru@NGT & 0.428 & $1 \mathrm{M} \mathrm{KOH}$ & 60 & This work \\
\hline Ru@NGT/NF & 0.5 & $1 \mathrm{M} \mathrm{KOH}$ & 45 & This work \\
\hline
\end{tabular}




\section{References}

1. Guo, J.; Gadipelli, S.; Yang, Y.; Li, Z.; Lu, Y.; Brett, D. J. L.; Guo, Z., An efficient carbon-based ORR catalyst from low-temperature etching of ZIF-67 with ultra-small cobalt nanoparticles and high yield. J. Mater. Chem. A 2019, 7 (8), 3544-3551.

2. Tang, Z.; Wu, W.; Wang, K. Oxygen Reduction Reaction Catalyzed by Noble Metal Clusters. Catalysts 2018, 8, 65

3. Huang, X.; Wang, J.; Bao, H.; Zhang, X.; Huang, Y., 3D Nitrogen, Sulfur-Codoped Carbon Nanomaterial-Supported Cobalt Oxides with Polyhedron-Like Particles Grafted onto Graphene Layers as Highly Active Bicatalysts for Oxygen-Evolving Reactions. ACS Appl. Mater. Interfaces 2018, 10 (8), 7180-7190.

4. Yu, Y.; Xiao, D.; Ma, J.; Chen, C.; Li, K.; Ma, J.; Liao, Y.; Zheng, L.; Zuo, X., The self-template synthesis of highly efficient hollow structure $\mathrm{Fe} / \mathrm{N} / \mathrm{C}$ electrocatalysts with $\mathrm{Fe}-\mathrm{N}$ coordination for the oxygen reduction reaction. RSC Adv. 2018, 8 (43), 24509-24516

5. Zhu, Q.-L.; Xia, W.; Zheng, L.-R.; Zou, R.; Liu, Z.; Xu, Q., Atomically Dispersed Fe/N-Doped Hierarchical Carbon Architectures Derived from a Metal-Organic Framework Composite for Extremely Efficient Electrocatalysis. ACS Energy Lett. 2017, 2 (2), 504-511.

6. Liu, S.; Mu, X.; Duan, H.; Chen, C.; Zhang, H., Pd Nanoparticle Assemblies as Efficient Catalysts for the Hydrogen Evolution and Oxygen Reduction Reactions. European Journal of Inorg. Chem. 2017, 2017 (3), 535-539.

7. Tran, T.-N.; Song, M. Y.; Singh, K. P.; Yang, D.-S.; Yu, J.-S., Iron-polypyrrole electrocatalyst with remarkable activity and stability for ORR in both alkaline and acidic conditions: a comprehensive assessment of catalyst preparation sequence. J. Mater. Chem. A 2016, 4 (22), 8645-8657.

8. Xiao, C.; Chen, X.; Fan, Z.; Liang, J.; Zhang, B.; Ding, S., Surface-nitrogen-rich ordered mesoporous carbon as an efficient metal-free electrocatalyst for oxygen reduction reaction. Nanotechnology 2016, 27 (44), 445402.

9. Ferrero, G. A.; Preuss, K.; Fuertes, A. B.; Sevilla, M.; Titirici, M. M., The influence of pore size distribution on the oxygen reduction reaction performance in nitrogen doped carbon microspheres. J. Mater. Chem. A 2016, 4 (7), 2581-2589.

10. Aijaz, A.; Masa, J.; Rösler, C.; Xia, W.; Weide, P.; Botz, A. J. R.; Fischer, R. A.; Schuhmann, W.; Muhler, M., Co@Co3O4 Encapsulated in Carbon Nanotube-Grafted Nitrogen-Doped Carbon Polyhedra as an Advanced Bifunctional Oxygen Electrode. Angew. Chem. Int. Ed. 2016, 55 (12), 4087-4091.

11. Shang, L.; Yu, H.; Huang, X.; Bian, T.; Shi, R.; Zhao, Y.; Waterhouse, G. I. N.; Wu, L.-Z.; Tung, C.-H.; Zhang, T., Well-Dispersed ZIF-Derived Co,N-Co-doped Carbon Nanoframes through Mesoporous-Silica-Protected Calcination as Efficient Oxygen Reduction Electrocatalysts. Adv. Mater. 2016, 28 (8), 1668-1674. 
12. Xi, J.; Xia, Y.; Xu, Y.; Xiao, J.; Wang, S., (Fe,Co)@ nitrogen-doped graphitic carbon nanocubes derived from polydopamine-encapsulated metal-organic frameworks as a highly stable and selective non-precious oxygen reduction electrocatalyst. ChemComm 2015, 51 (52), 1047910482.

13. Wang, S.; Cui, Z.; Cao, M., A Template-Free Method for Preparation of Cobalt Nanoparticles Embedded in N-Doped Carbon Nanofibers with a Hierarchical Pore Structure for Oxygen Reduction. Chem.: Eur. J. 2015, 21 (5), 2165-2172.

14. Zhang, L.; Wang, X.; Wang, R.; Hong, M., Structural Evolution from Metal-Organic Framework to Hybrids of Nitrogen-Doped Porous Carbon and Carbon Nanotubes for Enhanced Oxygen Reduction Activity. Chem. Mater. 2015, 27 (22), 7610-7618.

15. Lai, Q.; Su, Q.; Gao, Q.; Liang, Y.; Wang, Y.; Yang, Z.; Zhang, X.; He, J.; Tong, H., In Situ SelfSacrificed Template Synthesis of Fe-N/G Catalysts for Enhanced Oxygen Reduction. ACS Appl. Mater. Interfaces 2015, 7 (32), 18170-18178.

16. Zhang, X.; Liang, Y., Nickel Hydr(oxy)oxide Nanoparticles on Metallic MoS2 Nanosheets: A Synergistic Electrocatalyst for Hydrogen Evolution Reaction. Adv. Sci. 2018, 5 (2), 1700644.

17. Zheng, Y.-R.; Wu, P.; Gao, M.-R.; Zhang, X.-L.; Gao, F.-Y.; Ju, H.-X.; Wu, R.; Gao, Q.; You, R.; Huang, W.-X.; Liu, S.-J.; Hu, S.-W.; Zhu, J.; Li, Z.; Yu, S.-H., Doping-induced structural phase transition in cobalt diselenide enables enhanced hydrogen evolution catalysis. Nat. Commun. 2018, 9 (1), 2533.

18. Zhu, Z.; Yin, H.; He, C.-T.; Al-Mamun, M.; Liu, P.; Jiang, L.; Zhao, Y.; Wang, Y.; Yang, H.-G.; Tang, Z.; Wang, D.; Chen, X.-M.; Zhao, H., Ultrathin Transition Metal Dichalcogenide/3d Metal Hydroxide Hybridized Nanosheets to Enhance Hydrogen Evolution Activity. Adv. Mater. 2018, 30 (28), 1801171.

19. Chen, W.; Pei, J.; He, C.-T.; Wan, J.; Ren, H.; Wang, Y.; Dong, J.; Wu, K.; Cheong, W.-C.; Mao, J.; Zheng, X.; Yan, W.; Zhuang, Z.; Chen, C.; Peng, Q.; Wang, D.; Li, Y., Single Tungsten Atoms Supported on MOF-Derived N-Doped Carbon for Robust Electrochemical Hydrogen Evolution. Adv. Mater. 2018, 30 (30), 1800396.

20. Feng, J.-X.; Wu, J.-Q.; Tong, Y.-X.; Li, G.-R., Efficient Hydrogen Evolution on Cu NanodotsDecorated Ni3S2 Nanotubes by Optimizing Atomic Hydrogen Adsorption and Desorption. J. Am. Chem. Soc. 2018, 140 (2), 610-617.

21. Ma, Y.-Y.; Wu, C.-X.; Feng, X.-J.; Tan, H.-Q.; Yan, L.-K.; Liu, Y.; Kang, Z.-H.; Wang, E.-B.; Li, Y.G., Highly efficient hydrogen evolution from seawater by a low-cost and stable CoMoP@C electrocatalyst superior to Pt/C. Energy Environ. Sci. 2017, 10 (3), 788-798.

22. Wu, Z.; Guo, J.; Wang, J.; Liu, R.; Xiao, W.; Xuan, C.; Xia, K.; Wang, D., Hierarchically Porous Electrocatalyst with Vertically Aligned Defect-Rich CoMoS Nanosheets for the Hydrogen Evolution Reaction in an Alkaline Medium. ACS Appl. Mater. Interfaces 2017, 9 (6), 5288-5294.

23. Hu, C.; Ma, Q.; Hung, S.-F.; Chen, Z.-N.; Ou, D.; Ren, B.; Chen, H. M.; Fu, G.; Zheng, N., In Situ Electrochemical Production of Ultrathin Nickel Nanosheets for Hydrogen Evolution Electrocatalysis. Chem 2017, 3 (1), 122-133. 
24. Liu, B.; Zhao, Y.-F.; Peng, H.-Q.; Zhang, Z.-Y.; Sit, C.-K.; Yuen, M.-F.; Zhang, T.-R.; Lee, C.-S.; Zhang, W.-J., Nickel-Cobalt Diselenide 3D Mesoporous Nanosheet Networks Supported on Ni Foam: An All-pH Highly Efficient Integrated Electrocatalyst for Hydrogen Evolution. Adv. Mater. 2017, 29 (19), 1606521.

25. Yu, L.; Zhou, H.; Sun, J.; Qin, F.; Yu, F.; Bao, J.; Yu, Y.; Chen, S.; Ren, Z., Cu nanowires shelled with NiFe layered double hydroxide nanosheets as bifunctional electrocatalysts for overall water splitting. Energy Environ. Sci. 2017, 10 (8), 1820-1827.

26. Qu, K.; Zheng, Y.; Zhang, X.; Davey, K.; Dai, S.; Qiao, S. Z., Promotion of Electrocatalytic Hydrogen Evolution Reaction on Nitrogen-Doped Carbon Nanosheets with Secondary Heteroatoms. ACS Nano 2017, 11 (7), 7293-7300.

27. Yoon, D.; Lee, J.; Seo, B.; Kim, B.; Baik, H.; Joo, S. H.; Lee, K., Cactus-Like Hollow Cu2-xS@Ru Nanoplates as Excellent and Robust Electrocatalysts for the Alkaline Hydrogen Evolution Reaction. Small 2017, 13 (29), 1700052.

28. Zheng, Y.; Jiao, Y.; Zhu, Y.; Li, L. H.; Han, Y.; Chen, Y.; Jaroniec, M.; Qiao, S.-Z., High Electrocatalytic Hydrogen Evolution Activity of an Anomalous Ruthenium Catalyst. J. Am. Chem. Soc. 2016, 138 (49), 16174-16181.

29. Bhowmik, T.; Kundu, M. K.; Barman, S., Growth of One-Dimensional RuO2 Nanowires on gCarbon Nitride: An Active and Stable Bifunctional Electrocatalyst for Hydrogen and Oxygen Evolution Reactions at All pH Values. ACS Appl. Mater. Interfaces 2016, 8 (42), 28678-28688.

30. Tang, C.; Cheng, N.; Pu, Z.; Xing, W.; Sun, X., NiSe Nanowire Film Supported on Nickel Foam: An Efficient and Stable 3D Bifunctional Electrode for Full Water Splitting. Angew. Chem. Int. Ed. 2015, 54 (32), 9351-9355. 